Original Article

\title{
Aging effects on the diurnal patterns of gut microbial composition in male and female mice
}

\author{
Hyun-Jung Kim ${ }^{1}$, Chang Mo Moon ${ }^{2,3}$, Jihee Lee Kang ${ }^{3,4, *}$, and Eun-Mi Park ${ }^{1, *}$ \\ Departments of ${ }^{1}$ Pharmacology and ${ }^{2}$ Internal Medicine, College of Medicine, Ewha Womans University, ${ }^{3}$ Inflammation-Cancer Microenvironment Research \\ Center, College of Medicine, Ewha Womans University, ${ }^{4}$ Department of Physiology, College of Medicine, Ewha Womans University, Seoul 07084, Korea
}

\author{
ARTICLE INFO \\ Received June 9, 2021 \\ Revised September 7, 2021 \\ Accepted September 7, 2021 \\ *Correspondence \\ Jihee Lee Kang \\ E-mail: jihee@ewha.ac.kr \\ Eun-Mi Park \\ E-mail:empark@ewha.ac.kr
}

Key Words

Aging

Diurnal rhythm

Dysbiosis

Gut microbiota

Sex difference
ABSTRACT Composition of the gut microbiota changes with aging and plays an important role in age-associated disease such as metabolic syndrome, cancer, and neurodegeneration. The gut microbiota composition oscillates through the day, and the disruption of their diurnal rhythm results in gut dysbiosis leading to metabolic and immune dysfunctions. It is well documented that circadian rhythm changes with age in several biological functions such as sleep, body temperature, and hormone secretion. However, it is not defined whether the diurnal pattern of gut microbial composition is affected by aging. To evaluate aging effects on the diurnal pattern of the gut microbiome, we evaluated the taxa profiles of cecal contents obtained from young and aged mice of both sexes at daytime and nighttime points by $16 \mathrm{~S}$ rRNA gene sequencing. At the phylum level, the ratio of Firmicutes to Bacteroidetes and the relative abundances of Verrucomicrobia and Cyanobacteria were increased in aged male mice at night compared with that of young male mice. Meanwhile, the relative abundances of Sutterellaceae, Alloprevotella, Lachnospiraceae UCG-001, and Parasutterella increased in aged female mice at night compared with that of young female mice. The Lachnospiraceae NK4A136 group relative abundance increased in aged mice of both sexes but at opposite time points. These results showed the changes in diurnal patterns of gut microbial composition with aging, which varied depending on the sex of the host. We suggest that disturbed diurnal patterns of the gut microbiome can be a factor for the underlying mechanism of age-associated gut dysbiosis.

\section{INTRODUCTION}

The microbiota of the gut influences many aspects of host physiology and biological functions, such as mucosal integrity of the intestinal epithelial barrier, food digestion and absorption, and production of neurotransmitters and hormones [1]. The gut microbiota, which is dominated by bacteria, also plays an important role in a wide range of diseases beyond the intestine, such as metabolic syndrome and cancer [1]. For example, in the intestine of subjects with metabolic syndrome, the relative abundance of two dominant phyla, Firmicutes and Bacteroidetes, are changed and bacterial diversity is reduced (gut dysbiosis). A causal role of gut microbiota in developing metabolic diseases has been further defined by gut microbiota transplantation, which replicates the phenotype of metabolic syndrome [2-4].

The gut microbiota also changes with aging. Specifically, the abundance of normally dominant bacteria decreases while opportunistic pathobionts increases and the diversity of the gut microbiota decreases in the gut of aged subjects [5]. These agerelated changes in gut microbiota are implicated in developing low-grade chronic inflammation (inflammaging), which is a risk factor for morbidity and mortality in the elderly [6]. In addition, (i) \$ This is an Open Access article distributed under the terms of the Creative Commons Attribution Non-Commercial License, which permits unrestricted non-commercial use, distribution, and reproduction in any medium, provided the original work is properly cited. Copyright @ Korean J Physiol Pharmacol, pISSN 1226-4512, eISSN 2093-3827
Author contributions: H.J.K.: Acquisition of data, analysis of data, drafting the manuscript. C.M.M.: Design of study, interpretation of data. J.L.K.: Conception of study, interpretation of data, drafting the manuscript. E.M.P.: Conception of design of study, interpretation of data, drafting the manuscript. 
recent studies showed the association between age-related gut dysbiosis and the pathogenesis of atherosclerosis, type 2 diabetes, and neurodegeneration that are prevalent in the elderly [7-10]. Contributors to age-related changes in the gut microbiota include leaky gut, Western diet, changes in nutrient intake, medications, and increased hypothalamus-pituitary-adrenal axis activity $[11,12]$. However, a causal relationship and the underlying mechanism of age-related gut dysbiosis are not still clearly defined.

Circadian rhythms are present in most organisms and synchronize important physiological activities such as sleep/wake, hormone release, and metabolism [13]. A master clock in the suprachiasmatic nucleus of the hypothalamus regulates rhythmicity of physiology, and the environmental light/dark cycle serves as a the strongest signal to synchronize circadian rhythms [14]. It has been reported that the abundance of certain gut bacteria also oscillates rhythmically during the course of a day [15-17]. For example, the proportion of the phylum Firmicutes in the gut peaks during dark phase (active phase) and bottoms during light phase (rest phase). Meanwhile, the proportions of the phyla Bacteroidetes and Verrucomicrobia exhibit antiphasic patterns. The diurnal rhythm of gut microbiota is disrupted by molecular clock gene deficiency, high fat diet, food restriction, and shifts in the light/ dark cycle, which influence the host circadian physiology and the susceptibility to metabolic diseases [15-19]. These studies indicate that microbial rhythm is interrelated to the host circadian rhythm. In general, as the circadian rhythm declines with aging $[20,21]$, it can be assumed that the diurnal composition of the gut microbiota may also change with aging. However, their diurnal patterns in aged subjects compared with those of young subjects has not been determined.

We previously reported that 20-month-old mice of both sexes are reproductively senescent and exhibit increased visceral adiposity, a feature of aging, compared with young 10-12 weeks-old mice $[22,23]$. In this study, to evaluate the effects of aging on the diurnal patterns of the gut microbiota composition, we compared the composition of gut microbiota in the cecum of young and aged male and female young and aged mice sampled at two different time points of light/dark periods.

\section{METHODS}

\section{Animals and sample collection}

Male and female C57BL/6 mice (6 weeks old) were purchased from Orient Bio Inc. (Seongnam, Korea) and maintained in a specific pathogen-free animal facility at Ewha Womans University Medical College on a 12-h light/dark cycle (8:00 AM, light on; 8:00 PM, light off) at $22 \pm 2^{\circ} \mathrm{C}$. It has been reported that mice fed normal chow ad libitum exhibit cyclical fluctuations in phyla within the gut microbiome [15]. Therefore, in the present study, mice were given standard rodent chow, and tap water ad libitum.
Experiments were performed using young adult mice (2.5 months old), and aged mice (20 months old). Three mice from different cages were used for gut microbiota analysis, and therefore, six young (body weights of male and female were $25.50 \pm 0.48 \mathrm{~g}$ and $21.17 \pm 0.48$ g, respectively) and six aged (body weights of male and female mice were $38.37 \pm 1.53 \mathrm{~g}$ and $28.67 \pm 0.71 \mathrm{~g}$, respectively) mice of each sex were used ( $\mathrm{n}=3$ per sampling time point). The cecum of the mice was collected under terminal sodium pentobarbital anesthesia $(120 \mathrm{mg} / \mathrm{kg}$, intraperitoneal) at $10 \mathrm{AM}$ for the light period of day (AM) and $10 \mathrm{PM}$ for the dark period of night (PM, Fig. 1A). For the PM sampling, the mice were anesthetized in the dark and the cecum then obtained under light. The cecum specimens were stored at $-80^{\circ} \mathrm{C}$ until analyses. All procedures were approved by the Institutional Animal Care and Use Committee at the Medical School of Ewha Womans University (EWHA MEDIACUC past-057) and conformed to the international guidelines for the ethical use of experimental animals.

\section{Bacterial DNA extraction and 165 ribosomal RNA gene sequencing}

DNA of the cecal contents was extracted using a DNA Fast Stool Mini Kit (Qiagen, Hilden, Germany) according to the manufacturer's protocol. The $16 \mathrm{~S}$ ribosomal RNA (rRNA) gene analysis was performed at Ebiogen Inc. (Seoul, Korea), as previously described [24]. Briefly, each sequenced sample was prepared according to the Illumina 16S Metagenomic Sequencing Library protocols and the genes were amplified using 16S V3-V4 primers. The final products were normalized, pooled, and the size of the libraries verified using the Agilent TapeStation DNA ScreenTape D1000 system (Agilent Technologies, Santa Clara, CA, USA). Finally, the pooled libraries were sequenced $(2 \times 300)$ using the MiSeq platform (Illumina, San Diego, CA, USA). The amplicon error was modelled from paired fastq using DADA2 (ver.1.10.1) and filtered out noise sequence, corrected errors in marginal sequences, removed chimeric sequences and singleton, and then dereplicated those sequences. Each taxon level was classified based on the SILVA rRNA (region V3-V4) reference database (https://www.arb-silva.de/) using a q2-feature classifier. Each diversity metric was calculated by a q2-diversity program. The percent of total reads was calculated for each mouse and then averaged per sampling time point.

\section{Statistical analysis}

Data are expressed as the mean \pm standard error of the mean (SEM). Differences between groups were analyzed using KruskalWallis followed by the Dunn's test. Prism9.0 software (GraphPad Software, Inc., La Jolla, CA, USA) was used to perform the statistical analyses. Statistical significance was set at $\mathrm{p}<0.05$. 

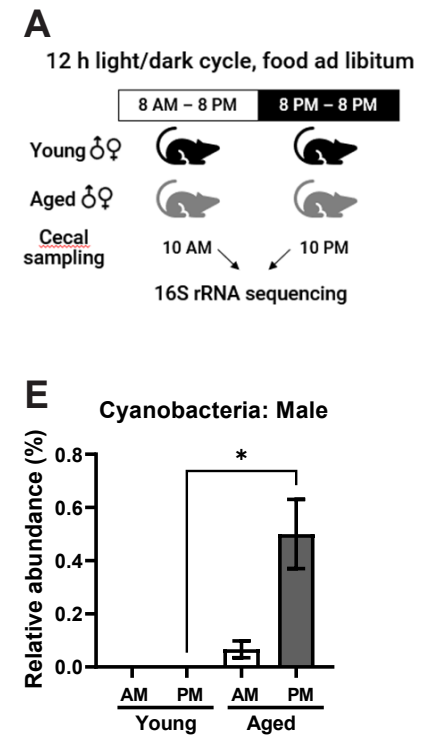

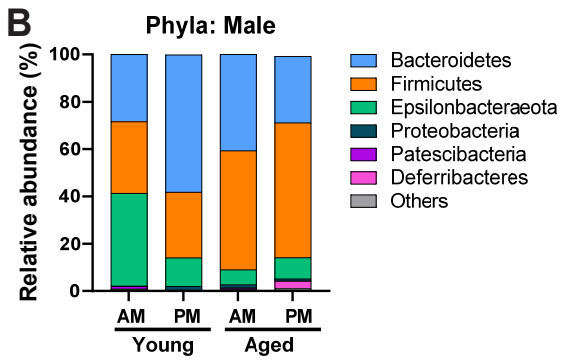

$\mathbf{F}$

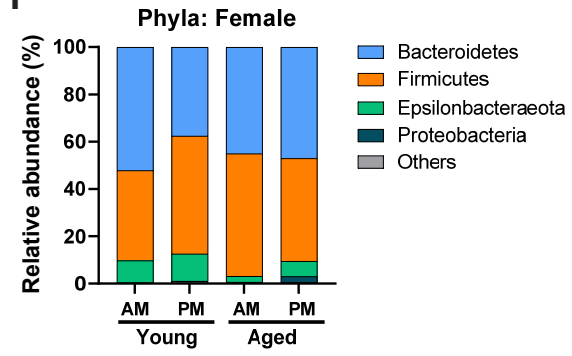

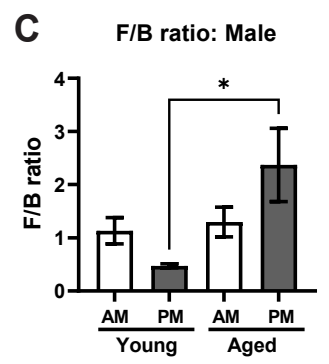

G

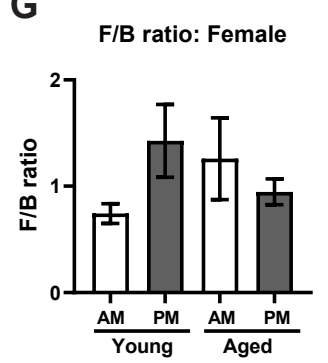

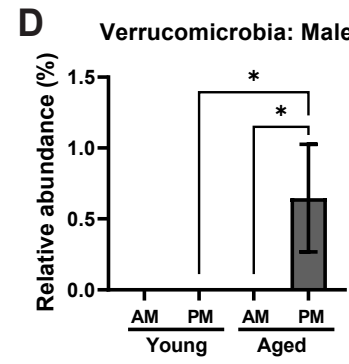

H

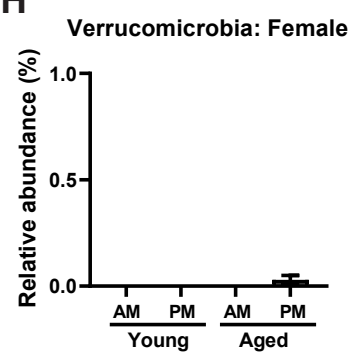

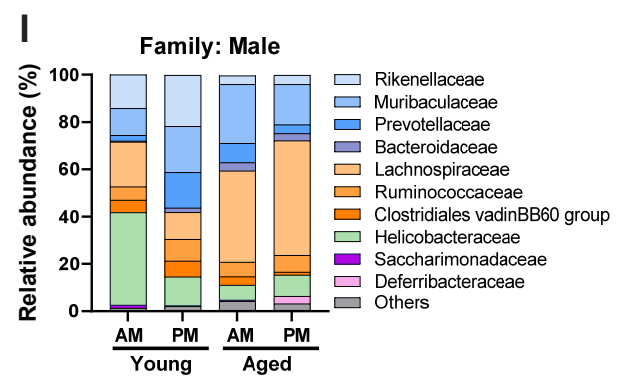
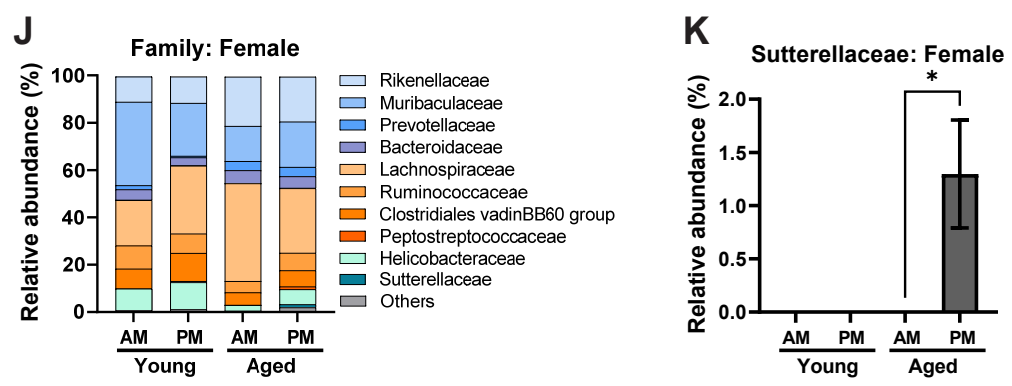

Fig. 1. Diurnal differences of the gut microbiota between young and aged mice at the phylum and family levels. (A) Experimental scheme of gut sampling times in young and aged mice according to light/dark cycle. (B-E) Mean gut microbiota values of cecum collected at 10 AM (AM) and 10 PM (PM) samples of young and aged male mice at the phylum level. (B) Stacked bar chart of microbiota composition of phyla present at $>1 \%$ relative abundance of male mice. (C) Firmicutes to Bacteroidetes (F/B) ratio of male mice. (D) Relative abundance of Verrucomicrobia of male mice. (E) Relative abundance of Cyanobacteria of male mice. (F) Stacked bar chart of microbiota composition of phyla present at $>1 \%$ relative abundance of females. (G) F/B ratio of female mice. $(\mathrm{H})$ Relative abundance of Verrucomicrobia of female mice. (I-K) Mean gut microbiota values of AM and PM cecum samples of young and aged mice at the family level. (I) Stacked bar chart of microbiota composition of families present at $>1 \%$ relative abundance of male mice. (J) Stacked bar chart of microbiota composition of families present at $>1 \%$ relative abundance of female mice. (K) Relative abundance of Sutterellaceae of female mice. $n=3 /$ group, ${ }^{*} p<0.05$.

\section{RESULTS}

\section{Diurnal changes of gut microbiota composition with aging at the phylum and family levels}

The number of operational taxonomic units (OTUs) and diversity, including alpha-diversity (intrasample diversity), betadiversity (intersample diversity), and evenness, were compared between AM and PM samples of young and aged mice to determine diurnal patterns in general characteristics of gut microbiota with aging. We found no statistical differences between the AM and PM samples in either young or aged mice of either sex for any of either sex for any of the parameters investigated (Table 1). Also, no statistical changes were observed between young and aged mice at neither AM nor PM, although the number of OTUs in both AM and PM decreased in aged mice compared to those of young mice. These results are consistent with a previous study that reported decreased bacterial richness and diversity without statistical significance in cecal samples from aged mice compared to those of young mice [25].

At the phylum level, there were also no changes with aging of the diurnal patterns of bacteria with more than $1 \%$ relative abundance, regardless of sex (Table 2, Fig. 1B, F). However, the ratio of Firmicutes to Bacteroidetes ( $\mathrm{F} / \mathrm{B}$ ratio), which is indicative of dysbiosis [26], was significantly increased in aged male mice compared with that of young male mice for the PM samples ( $p=0.04$; Fig. 1C). The relative abundance of the minor phyla Verrucomicrobia and Cyanobacteria were also increased in aged male mice compared with that of young male mice for the PM samples (both $p=0.04$; Fig. 1D, E). Meanwhile, there were no diurnal changes in F/B ratios between young and aged female mice (Fig. 1G). In addition, the relative abundance of Verrucomicrobia was slightly in- 
Table 1. Characteristics of metagenome sequencing data and diversity of microbiome from cecal contents of male and female mice

\begin{tabular}{|c|c|c|c|c|c|}
\hline \multirow{2}{*}{ Characteristics } & \multicolumn{2}{|c|}{ Young } & \multicolumn{2}{|c|}{ Aged } & \multirow{2}{*}{$\mathrm{p}$-value } \\
\hline & AM & PM & $\mathrm{AM}$ & PM & \\
\hline \multicolumn{6}{|l|}{ Male } \\
\hline No. of OTUs & $317.0 \pm 10.97$ & $363.3 \pm 17.89$ & $289.7 \pm 16.37$ & $320.3 \pm 39.07$ & 0.30 \\
\hline Alpha diversity (Shannon Index) & $4.83 \pm 0.26$ & $5.38 \pm 0.10$ & $6.30 \pm 0.11$ & $4.91 \pm 0.73$ & 0.16 \\
\hline Beta diversity & $0.51 \pm 0.03$ & $0.61 \pm 0.04$ & $0.52 \pm 0.06$ & $0.57 \pm 0.05$ & 0.36 \\
\hline Faith's phylogenetic diversity & $18.08 \pm 2.49$ & $23.51 \pm 2.63$ & $18.71 \pm 2.59$ & $23.51 \pm 6.49$ & 0.67 \\
\hline Evenness (Peilou Index) & $0.58 \pm 0.03$ & $0.63 \pm 0.01$ & $0.77 \pm 0.01$ & $0.59 \pm 0.07$ & 0.05 \\
\hline \multicolumn{6}{|l|}{ Female } \\
\hline No. of OTUs & $387.3 \pm 30.37$ & $398.7 \pm 17.61$ & $252.0 \pm 10.00$ & $361.7 \pm 11.20$ & 0.08 \\
\hline Alpha diversity (Shannon Index) & $6.17 \pm 0.28$ & $6.05 \pm 0.06$ & $4.43 \pm 0.04$ & $5.95 \pm 0.16$ & 0.07 \\
\hline Beta diversity & $0.43 \pm 0.00$ & $0.40 \pm 0.01$ & $0.31 \pm 0.01$ & $0.48 \pm 0.08$ & 0.10 \\
\hline Faith's phylogenetic diversity & $19.76 \pm 1.16$ & $19.89 \pm 0.45$ & $15.85 \pm 0.43$ & $23.88 \pm 3.98$ & 0.07 \\
\hline Evenness (Peilou Index) & $0.72 \pm 0.024$ & $0.70 \pm 0.00$ & $0.56 \pm 0.01$ & $0.70 \pm 0.02$ & 0.06 \\
\hline
\end{tabular}

Values are presented as mean \pm SEM. OTUs, operational taxonomic units.

Table 2. The diurnal relative abundance of gut microbiota at the phylum level in young and aged mice

\begin{tabular}{|c|c|c|c|c|c|}
\hline \multirow{2}{*}{ Phylum } & \multicolumn{2}{|c|}{ Young } & \multicolumn{2}{|c|}{ Aged } & \multirow{2}{*}{ p-value } \\
\hline & AM & PM & AM & PM & \\
\hline \multicolumn{6}{|l|}{ Male } \\
\hline Bacteroidetes & $28.47 \pm 5.33$ & $58.10 \pm 1.70$ & $40.67 \pm 4.61$ & $28.27 \pm 7.15$ & 0.08 \\
\hline Firmicutes & $30.17 \pm 5.83$ & $27.70 \pm 2.70$ & $50.30 \pm 4.53$ & $57.27 \pm 3.98$ & 0.08 \\
\hline Epsilonbacteraeota & $39.17 \pm 8.21$ & $12.13 \pm 3.28$ & $6.30 \pm 1.86$ & $8.97 \pm 2.97$ & 0.08 \\
\hline Proteobacteria & $0.16 \pm 0.09$ & $1.57 \pm 0.92$ & $1.30 \pm 0.32$ & $0.90 \pm 0.10$ & 0.05 \\
\hline Patescibacteria & $1.24 \pm 1.14$ & $0.30 \pm 0.06$ & $0.50 \pm 0.06$ & $0.03 \pm 0.03$ & 0.09 \\
\hline Deferribacteres & $0.01 \pm 0.01$ & $0.01 \pm 0.01$ & $0.11 \pm 0.09$ & $3.08 \pm 1.91$ & 0.23 \\
\hline \multicolumn{6}{|l|}{ Female } \\
\hline Bacteroidetes & $52.13 \pm 3.77$ & $37.54 \pm 5.49$ & $44.96 \pm 6.23$ & $47.01 \pm 4.26$ & 0.32 \\
\hline Firmicutes & $38.09 \pm 2.59$ & $49.84 \pm 5.79$ & $51.85 \pm 7.18$ & $43.50 \pm 2.52$ & 0.21 \\
\hline Epsilonbacteraeota & $9.36 \pm 2.53$ & $11.47 \pm 0.68$ & $2.50 \pm 1.24$ & $6.37 \pm 3.47$ & 0.13 \\
\hline Proteobacteria & $0.04 \pm 0.01$ & $0.35 \pm 0.51$ & $0.59 \pm 1.15$ & $2.52 \pm 0.56$ & 0.33 \\
\hline
\end{tabular}

Values are presented as mean \pm SEM. Phyla listed at $>1 \%$ relative abundance.

creased in aged female mice at PM, but no statistical significance was observed between young and aged female mice (Fig. 1H). Unlike the results of male mice, Cyanobacteria were not detected in either young or aged female mice.

At the family level, differences in diurnal patterns of bacteria with more than $1 \%$ relative abundance were not observed between young and aged mice in either sex (Table 3, Fig. 1I, J), except for the family Sutterellaceae that was not observed in male mice. The relative abundance of Sutterellaceae increased in aged female mice compared with that in young female mice at PM ( $\mathrm{p}$ $=0.04$; Fig. $1 \mathrm{~K}$ ). These findings indicate that the diurnal patterns of some components of the gut microbiome at phylum and family levels change with aging and there are sex-associated dissimilarities.

\section{Diurnal changes of gut microbiota composition with aging at the genus level}

The gut microbiome changes at genus level with aging $[5,25]$, and therefore we determined diurnal patterns of bacteria genera present at $>1 \%$ abundance.

In male mice, four bacteria showed differences in their diurnal patterns. In young male mice, the relative abundances of Rikenella and Rikenellaceae RC9 gut group of the phylum Bacteroidetes were significantly increased in AM samples compared with those in $\mathrm{PM}(\mathrm{p}=0.04$ and $\mathrm{p}=0.01$, respectively); however, their relative abundances were decreased in aged male mice at both time points (Fig. 2A, B). The significant decrease in the relative abundance of Rikenella in aged male mice compared with that in young male mice was especially observed in AM samples $(\mathrm{p}=0.04$; Fig. $2 \mathrm{~A})$. The relative abundance of Lachnospiraceae NK4A136 group of the phylum Firmicutes was markedly increased in aged male mice compared with that in young male mice and the increase 

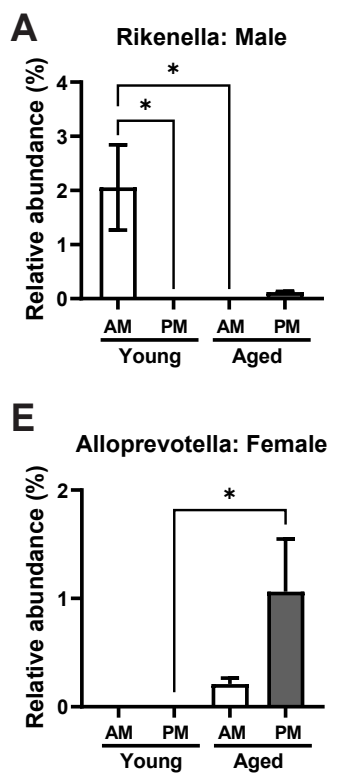
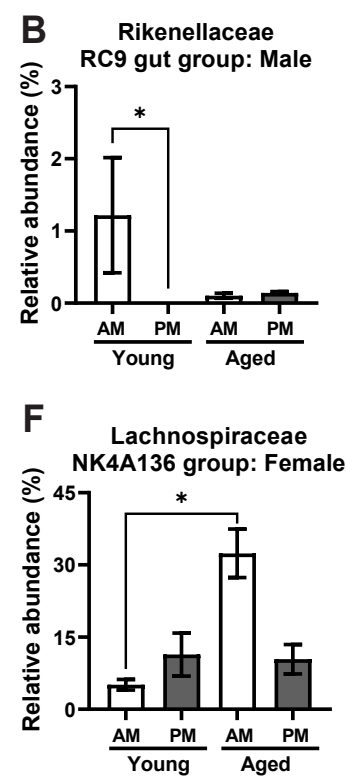
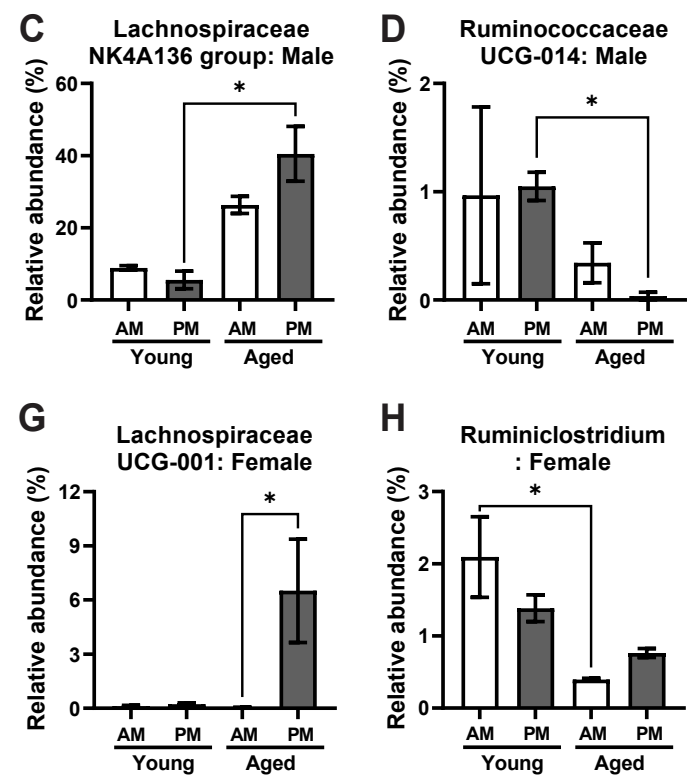

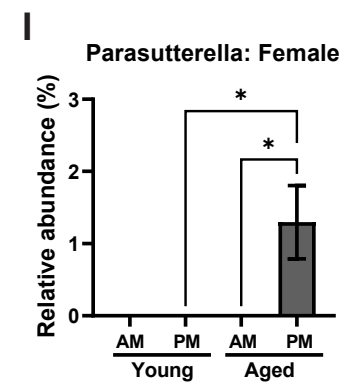

Fig. 2. Diurnal differences of gut microbiota between young and aged mice at the genus level. (A-D) Mean gut microbiota values of cecum collected at 10 AM (AM) and 10 PM (PM) samples of young and aged male mice. (A) Relative abundance of Rikenella. (B) Relative abundance of Rikenellaceae RC9 gut group. (C) Relative abundance of Lachnospiraceae NK4A136 group. (D) Relative abundance of Ruminococcaceae UCG-014. (E-I) Mean gut microbiota values of AM and PM cecum samples of young and aged female mice. (E) Relative abundance of Alloprevotella. (F) Relative abundance of Lachnospiraceae NK4A136 group. (G) Relative abundance of Lachnospiraceae UCG-001. (H) Relative abundance of Ruminiclostridium. (I) Relative abundance of Parasutterella. $\mathrm{n}=3 /$ group, ${ }^{*} \mathrm{p}<0.05$.

Table 3. The diurnal relative abundance of gut microbiota at the family level in young and aged mice

\begin{tabular}{|c|c|c|c|c|c|}
\hline \multirow{2}{*}{ Family } & \multicolumn{2}{|c|}{ Young } & \multicolumn{2}{|c|}{ Aged } & \multirow{2}{*}{ p-value } \\
\hline & AM & PM & AM & PM & \\
\hline \multicolumn{6}{|l|}{ Male } \\
\hline Rikenellaceae & $14.20 \pm 6.35$ & $21.57 \pm 5.64$ & $3.60 \pm 0.86$ & $3.93 \pm 0.45$ & 0.11 \\
\hline Muribaculaceae & $11.37 \pm 3.50$ & $19.47 \pm 2.86$ & $24.83 \pm 2.28$ & $17.00 \pm 4.54$ & 0.12 \\
\hline Prevotellaceae & $2.50 \pm 2.11$ & $15.07 \pm 1.30$ & $8.17 \pm 2.86$ & $3.77 \pm 2.13$ & 0.06 \\
\hline Bacteroidaceae & $0.40 \pm 0.12$ & $1.97 \pm 0.37$ & $3.53 \pm 0.95$ & $3.07 \pm 0.95$ & 0.05 \\
\hline Lachnospiraceae & $18.87 \pm 4.78$ & $11.30 \pm 3.38$ & $38.58 \pm 4.10$ & $48.45 \pm 6.13$ & 0.08 \\
\hline Ruminococcaceae & $5.69 \pm 1.62$ & $9.20 \pm 0.99$ & $6.17 \pm 1.64$ & $7.04 \pm 2.01$ & 0.61 \\
\hline Clostridiales vadin BB60 group & $5.22 \pm 2.01$ & $6.72 \pm 0.79$ & $3.50 \pm 1.42$ & $1.27 \pm 0.29$ & 0.24 \\
\hline Helicobacteraceae & $39.16 \pm 8.22$ & $12.13 \pm 3.28$ & $6.29 \pm 1.87$ & $8.97 \pm 2.99$ & 0.08 \\
\hline Saccharimonadaceae & $1.24 \pm 1.13$ & $0.32 \pm 0.06$ & $0.50 \pm 0.04$ & $0.05 \pm 0.05$ & 0.06 \\
\hline Deferribacteraceae & $0.01 \pm 0.01$ & $0.01 \pm 0.01$ & $0.11 \pm 0.09$ & $3.08 \pm 1.91$ & 0.23 \\
\hline \multicolumn{6}{|l|}{ Female } \\
\hline Rikenellaceae & $10.63 \pm 6.78$ & $11.16 \pm 1.91$ & $20.85 \pm 3.48$ & $18.94 \pm 1.95$ & 0.24 \\
\hline Muribaculaceae & $35.37 \pm 2.84$ & $22.29 \pm 1.88$ & $14.78 \pm 4.71$ & $19.19 \pm 5.43$ & 0.08 \\
\hline Prevotellaceae & $1.61 \pm 0.06$ & $0.64 \pm 0.33$ & $3.89 \pm 1.12$ & $3.94 \pm 1.72$ & 0.05 \\
\hline Bacteroidaceae & $4.47 \pm 0.26$ & $3.36 \pm 2.39$ & $5.43 \pm 1.11$ & $4.90 \pm 0.88$ & 0.86 \\
\hline Lachnospiraceae & $19.10 \pm 3.56$ & $28.76 \pm 4.39$ & $41.38 \pm 7.42$ & $27.34 \pm 2.48$ & 0.05 \\
\hline Ruminococcaceae & $9.85 \pm 1.67$ & $8.18 \pm 0.56$ & $4.70 \pm 0.37$ & $7.37 \pm 0.72$ & 0.06 \\
\hline Clostridiales vadin BB60 group & $8.34 \pm 1.14$ & $11.90 \pm 2.03$ & $5.40 \pm 1.081$ & $6.90 \pm 0.66$ & 0.09 \\
\hline Peptostreptococcaceae & 0 & $0.43 \pm 0.15$ & $0.01 \pm 0.01$ & $1.13 \pm 0.49$ & 0.15 \\
\hline Helicobacteraceae & $9.36 \pm 2.53$ & $11.47 \pm 0.68$ & $2.50 \pm 1.24$ & $6.37 \pm 3.47$ & 0.13 \\
\hline Sutterellaceae & 0 & 0 & 0 & $1.30 \pm 0.51$ & 0.04 \\
\hline
\end{tabular}

Values are presented as mean \pm SEM. Families listed at $>1 \%$ relative abundance.

was significant in PM samples ( $\mathrm{p}=0.04$; Fig. 2C). In contrast, the relative abundance of Ruminococcaceae UCG-014, which also belongs to the phylum Firmicutes, was decreased in aged male mice compared with that in young male mice, especially in PM samples ( $\mathrm{p}=0.04$; Fig. 2D).

In female mice, there were changes in the diurnal patterns 
of five bacteria, which differed from those of male mice. Alloprevotella and Parasutterella were not detected in young female mice, but were present at significant levels in aged female mice, especially in PM samples (both $\mathrm{p}=0.04$ vs. young female mice; Fig. 2E, I). The increased level of Parasutterella may have resulted from the increased relative abundance of the family Sutterellaceae (Fig. 1I). The relative abundance of Lachnospiraceae NK4A136 group was also significantly increased in aged female mice compared with that in young female mice in AM samples ( $p=0.02$; Fig. $2 \mathrm{~F}$ ), which was different from that observed in the aged male mice. The increased relative abundance of Lachnospiraceae UCG-001 was statistically significant in PM samples compared with AM samples of aged female mice ( $p=0.03$; Fig. $2 \mathrm{G}$ ). Meanwhile, the relative abundance of Ruminiclostridium, which also belongs to the phylum Firmicutes, decreased in aged female mice compared with young female mice, especially in AM samples ( $\mathrm{p}$ $=0.03$; Fig. $2 \mathrm{H}$ ). Taken together, these results indicate that the diurnal patterns of gut microbiota composition are affected at the genus level by aging with sex-associated dissimilarities.

\section{DISCUSSION}

The present study showed that the relative abundance of certain gut bacteria at several taxa levels changed with age between day and night times. In addition, the diurnal changes with aging in the gut microbiota composition were dissimilar between sexes. To the best of our knowledge, this is the first study to demonstrate diurnal changes in the relative abundance of some bacteria in the gut of aged mice of both sexes compared to young mice.

It has been reported that age-associated increase of $\mathrm{F} / \mathrm{B}$ ratio can be resulted from increased Firmicutes and/or decreased of Bacteroidetes abundances [25-28]. Interestingly, we observed that the significant increase in the $\mathrm{F} / \mathrm{B}$ ratio of aged male mice was only at nighttime. The increased $\mathrm{F} / \mathrm{B}$ ratio may have resulted from increased Firmicutes abundance, which was also reflected by the increased abundance of Lachnospiraceae NK4A136 group, which belongs to the phylum Firmicutes, at nighttime. Previous studies showed that the increase of $\mathrm{F} / \mathrm{B}$ ratio was linked to increases of lipopolysaccharide levels in the plasma and feces of aged male mice [25], and increased abundance of Cyanobacteria in mice with high $\mathrm{F} / \mathrm{B}$ ratio can be related to the severity of aging-associated dry eye [29]. We also observed the increases in F/B ratio and the relative abundance of Cyanobacteria in aged male mice at nighttime. Additionally, the relative abundance of Verrucomicrobia, which was increased in aged male mice at nighttime in the present study, has been positively correlated with plasma concentration of inflammatory cytokine interferon gamma in patients with Parkinson's disease [30]. Contrary to the results of male mice, neither the $\mathrm{F} / \mathrm{B}$ ratio nor microbiota composition at the phylum level showed any differences between young and aged female mice in the present study, which were comparable to a previous study reporting no changes in $\mathrm{F} / \mathrm{B}$ ratio of fecal samples collected from three different age groups (young, middle-aged, and old) [31]. Based on these previous studies, we suggest that altered diurnal patterns of $\mathrm{F} / \mathrm{B}$ ratio and abundance of Verrucomicrobia and Cyanobacteria have roles in inflammaging and severity of ageassociated diseases, at least in males.

At the family level, only the nighttime Sutterellaceae abundance differed significantly between young and aged female mice. The increase of Sutterellaceae abundance was again reflected by the increase of Parasutterella genus abundance in aged female mice at nighttime. A study reported that the abundance of Sutterellaceae increased in the feces of patients with irritable bowel syndrome [32], but the roles of Sutterellaceae and Parasutterella in aging and sex differences are remained to be determined. At genus level, more bacteria showed significant changes in diurnal patterns of their relative abundance in the gut of aged mice of both sexes, differentially. Lachnospiraceae NK4A136 group was dominant and their relative abundance markedly increased in the gut of aged mice of both sexes; however, the time points for the increase in their abundance were opposite in aged male (nighttime) and female (daytime) mice. It has been demonstrated the increase in Lachnospiraceae NK4A136 group abundance in mouse models of colitis and diet-induced obesity, suggesting pathological roles for these bacteria in diseases-associated inflammation $[33,34]$. The other genera such as Rikenella, Ruminococcaceae UCG-014, Alloprevotella, and Parasutterella, that showed changes in diurnal pattern of their abundance in the present study, have been also suggested to be involved in several diseases. Rikenella abundance decreased in the mouse gut of an Alzheimer's disease (AD) model, and gut-flora targeted photobiomodulation therapy reversed the changes in gut microbiome with improved cognitive function and brain pathologies [35]. The authors suggest that interventions targeting gut flora can be a treatment modality for $\mathrm{AD}$ [35]. Butyrate-producing Ruminococcaceae UCG-014 abundance also decreased in the mouse gut of a ulcerative colitis carcinogenesis model [36]. Meanwhile, the increases in the abundances of Alloprevotella and Parasutterella, which were also increased in aged female mice at nighttime in the present study, were observed in patients with irritable bowel syndrome [37,38], and Alloprevotella was one of the pathogens associated with chemotherapy-induced diarrhea in mice [39]. Furthermore, a recent study reported that ovariectomy, a model of menopause, induced osteoporosis and changed abundances of Alloprevotella and Parasutterella in the gut of female mice compared to the sham control, suggesting their roles in regulating bone homeostasis in postmenopausal women [40]. Considering that these taxa involved in the diseases with inflammation or aging, and that age-associated gut dysbiosis contributes to inflammaging [6], it is possible that diurnal changes in the abundance of certain gut bacteria may make the host vulnerable to diseases in which onset and symptoms worsen depending on the day or night. Typically, it is well known that osteoarthritis and Parkinson's disease symptoms are worse in the evening and 
night, and acute myocardial infarct incidents are most frequent in the morning [41]. However, the implications of aging-associated diurnal changes in the gut microbiota with respect to host health should be determined in further studies.

Sex differences in gut microbial composition, abundance, and diversity have been previously demonstrated, and both mice and human studies have shown the major roles of sex hormones in these difference [42-44]. Importantly, it has been suggested that sex-specific gut microbiome composition contributes to the development of sex-biased autoimmune diseases, cardiovascular diseases (CVD) and AD $[42,45,46]$. For example, the development of autoimmunity, prevalent in females, was reduced in nonobese diabetic female mice by gut microbiota transfer from adult male mice via the increase of testosterone level [42]. Sex differences in the prevalence of CVD and AD have been related with the differences in branched-chain amino acids and short-chain fatty acids derived from the gut microbiome $[45,46]$. Regarding a diurnal pattern of gut microbiota between sexes, only one study analyzed fecal pellets of young adult mice sampled every $4 \mathrm{~h}$ for $48 \mathrm{~h}$ [16]. The authors found that gut microbial rhythmicity in the composition during a day was more pronounced in young adult female mice than in male mice, and that the clock Bmall gene deletion altered the fecal microbial composition in a sex-dependent manner [16]. Although we did not directly compare the relative abundances of taxa between sexes, our results showed that diurnal patterns of gut microbiota composition differed according to the sex, of not only young but also aged mice. The rhythmicity and taxa in differences shown in the present study were not the same as those reported in the previous studies. These discrepancies may have resulted from differences in the experimental methods, such as sampling times (one-time sampling per light/dark period), intervals (sampling at $2 \mathrm{~h}$ after light turn on/off), and sites (cecum versus feces), as well as the small study size, which were also limitations of our study. As the focus of the present study was the aging effects on the diurnal pattern of gut microbiota, rather than sex differences in the gut microbiome, further studies to determine taxa differences during a day between sexes in aged subjects and their implications in host health are required.

In conclusion, we found that the relative abundance of some taxa at the phylum, family, and genus levels were changed according to light and dark periods in aged mice compared with those of young mice for both sexes. In addition, diurnal patterns and their changes in the gut microbiota with aging were dissimilar for males and females. We suggest that changes in the diurnal pattern of the gut microbiota composition contribute to age-associated gut dysbiosis with differences occurring between the sexes.

\section{ACKNOWLEDGEMENTS}

This work was supported by the intramural research program through the Ewha Education and Research for Infection funded by Ewha Womans University Medical Center (201900580001), Ewha Alumni Medical Research Grant, and the National Research Foundation of Korea (NRF) grant funded by the Korea government (MSIT) (NRF-2019R1A2C1087035 and NRF2020R1A5A2019210).

\section{CONFLICTS OF INTEREST}

The authors declare no conflicts of interest.

\section{REFERENCES}

1. Bishehsari F, Voigt RM, Keshavarzian A. Circadian rhythms and the gut microbiota: from the metabolic syndrome to cancer. Nat Rev Endocrinol. 2020;16:731-739.

2. Vijay-Kumar M, Aitken JD, Carvalho FA, Cullender TC, Mwangi S, Srinivasan S, Sitaraman SV, Knight R, Ley RE, Gewirtz AT. Metabolic syndrome and altered gut microbiota in mice lacking Toll-like receptor 5. Science. 2010;328:228-231.

3. Samuel BS, Shaito A, Motoike T, Rey FE, Backhed F, Manchester JK, Hammer RE, Williams SC, Crowley J, Yanagisawa M, Gordon JI. Effects of the gut microbiota on host adiposity are modulated by the short-chain fatty-acid binding $\mathrm{G}$ protein-coupled receptor, Gpr41. Proc Natl Acad Sci U S A. 2008;105:16767-16772.

4. Turnbaugh PJ, Ley RE, Mahowald MA, Magrini V, Mardis ER, Gordon JI. An obesity-associated gut microbiome with increased capacity for energy harvest. Nature. 2006;444:1027-1031.

5. Biagi E, Franceschi C, Rampelli S, Severgnini M, Ostan R, Turroni S, Consolandi C, Quercia S, Scurti M, Monti D, Capri M, Brigidi P, Candela M. Gut microbiota and extreme longevity. Curr Biol. 2016;26:1480-1485.

6. Rehman T. Role of the gut microbiota in age-related chronic inflammation. Endocr Metab Immune Disord Drug Targets. 2012;12:361367.

7. Karlsson FH, Fåk F, Nookaew I, Tremaroli V, Fagerberg B, Petranovic D, Bäckhed F, Nielsen J. Symptomatic atherosclerosis is associated with an altered gut metagenome. Nat Commun. 2012;3:1245.

8. Keshavarzian A, Green SJ, Engen PA, Voigt RM, Naqib A, Forsyth CB, Mutlu E, Shannon KM. Colonic bacterial composition in Parkinson's disease. Mov Disord. 2015;30:1351-1360.

9. Cattaneo A, Cattane N, Galluzzi S, Provasi S, Lopizzo N, Festari C, Ferrari C, Guerra UP, Paghera B, Muscio C, Bianchetti A, Volta GD, Turla M, Cotelli MS, Gennuso M, Prelle A, Zanetti O, Lussignoli G, Mirabile D, Bellandi D, et al. Association of brain amyloidosis with pro-inflammatory gut bacterial taxa and peripheral inflammation markers in cognitively impaired elderly. Neurobiol Aging. 2017;49:60-68.

10. Burcelin R, Serino M, Chabo C, Blasco-Baque V, Amar J. Gut microbiota and diabetes: from pathogenesis to therapeutic perspective. Acta Diabetol. 2011;48:257-273.

11. Buford TW. (Dis)Trust your gut: the gut microbiome in age-related inflammation, health, and disease. Microbiome. 2017;5:80.

12. DeJong EN, Surette MG, Bowdish DME. The gut microbiota and unhealthy aging: disentangling cause from consequence. Cell Host 
Microbe. 2020;28:180-189.

13. Mohawk JA, Green CB, Takahashi JS. Central and peripheral circadian clocks in mammals. Annu Rev Neurosci. 2012;35:445-462.

14. Schmalle V, Lorentz A. Role of the microbiota in circadian rhythms of the host. Chronobiol Int. 2020;37:301-310.

15. Zarrinpar A, Chaix A, Yooseph S, Panda S. Diet and feeding pattern affect the diurnal dynamics of the gut microbiome. Cell Metab. 2014;20:1006-1017

16. Liang X, Bushman FD, FitzGerald GA. Rhythmicity of the intestinal microbiota is regulated by gender and the host circadian clock. Proc Natl Acad Sci U S A. 2015;112:10479-10484.

17. Thaiss CA, Zeevi D, Levy M, Zilberman-Schapira G, Suez J, Tengeler AC, Abramson L, Katz MN, Korem T, Zmora N, Kuperman Y, Biton I, Gilad S, Harmelin A, Shapiro H, Halpern Z, Segal E, Elinav E. Transkingdom control of microbiota diurnal oscillations promotes metabolic homeostasis. Cell. 2014;159:514-529.

18. Leone V, Gibbons SM, Martinez K, Hutchison AL, Huang EY, Cham CM, Pierre JF, Heneghan AF, Nadimpalli A, Hubert N, Zale E, Wang Y, Huang Y, Theriault B, Dinner AR, Musch MW, Kudsk KA, Prendergast BJ, Gilbert JA, Chang EB. Effects of diurnal variation of gut microbes and high-fat feeding on host circadian clock function and metabolism. Cell Host Microbe. 2015;17:681-689.

19. Thaiss CA, Levy M, Korem T, Dohnalová L, Shapiro H, Jaitin DA, David E, Winter DR, Gury-BenAri M, Tatirovsky E, Tuganbaev T, Federici S, Zmora N, Zeevi D, Dori-Bachash M, Pevsner-Fischer M Kartvelishvily E, Brandis A, Harmelin A, Shibolet O, et al. Microbiota diurnal rhythmicity programs host transcriptome oscillations. Cell. 2016;167:1495-1510.e12.

20. Banks G, Nolan PM, Peirson SN. Reciprocal interactions between circadian clocks and aging. Mamm Genome. 2016;27:332-340.

21. Nakamura TJ, Nakamura W, Yamazaki S, Kudo T, Cutler T, Colwell CS, Block GD. Age-related decline in circadian output. J Neurosci. 2011;31:10201-10205

22. Shin JA, Jeong SI, Kim M, Yoon JC, Kim HS, Park EM. Visceral adipose tissue inflammation is associated with age-related brain changes and ischemic brain damage in aged mice. Brain Behav Immun. 2015;50:221-231.

23. Jeong SI, Shin JA, Cho S, Kim HW, Lee JY, Kang JL, Park EM. Resveratrol attenuates peripheral and brain inflammation and reduces ischemic brain injury in aged female mice. Neurobiol Aging. 2016;44:74-84

24. Crespo-Piazuelo D, Estellé J, Revilla M, Criado-Mesas L, RamayoCaldas Y, Óvilo C, Fernández AI, Ballester M, Folch JM. Characterization of bacterial microbiota compositions along the intestinal tract in pigs and their interactions and functions. Sci Rep. 2018;8:12727.

25. Kim KA, Jeong JJ, Yoo SY, Kim DH. Gut microbiota lipopolysaccharide accelerates inflamm-aging in mice. BMC Microbiol. 2016;16:9.

26. Spychala MS, Venna VR, Jandzinski M, Doran SJ, Durgan DJ, Ganesh BP, Ajami NJ, Putluri N, Graf J, Bryan RM, McCullough LD. Age-related changes in the gut microbiota influence systemic inflammation and stroke outcome. Ann Neurol. 2018;84:23-36.

27. Park SH, Kim KA, Ahn YT, Jeong JJ, Huh CS, Kim DH. Comparative analysis of gut microbiota in elderly people of urbanized towns and longevity villages. BMC Microbiol. 2015;15:49.

28. Hoffman JD, Parikh I, Green SJ, Chlipala G, Mohney RP, Keaton M, Bauer B, Hartz AMS, Lin AL. Age drives distortion of brain meta- bolic, vascular and cognitive functions, and the gut microbiome. Front Aging Neurosci. 2017;9:298.

29. Yoon CH, Ryu JS, Moon J, Kim MK. Association between aging-dependent gut microbiome dysbiosis and dry eye severity in C57BL/6 male mouse model: a pilot study. BMC Microbiol. 2021;21:106.

30. Lin CH, Chen CC, Chiang HL, Liou JM, Chang CM, Lu TP, Chuang EY, Tai YC, Cheng C, Lin HY, Wu MS. Altered gut microbiota and inflammatory cytokine responses in patients with Parkinson's disease. J Neuroinflammation. 2019;16:129.

31. Langille MG, Meehan CJ, Koenig JE, Dhanani AS, Rose RA, Howlett SE, Beiko RG. Microbial shifts in the aging mouse gut. Microbiome. 2014;2:50.

32. Lee SM, Kim N, Yoon H, Kim YS, Choi SI, Park JH, Lee DH. Compositional and functional changes in the gut microbiota in irritable bowel syndrome patients. Gut Liver. 2021;15:253-261.

33. Liu Y, Li T, Alim A, Ren D, Zhao Y, Yang X. Regulatory effects of stachyose on colonic and hepatic inflammation, gut microbiota dysbiosis, and peripheral CD4+ T cell distribution abnormality in highfat diet-fed mice. J Agric Food Chem. 2019;67:11665-11674.

34. Shao X, Sun C, Tang X, Zhang X, Han D, Liang S, Qu R, Hui X, Shan Y, Hu L, Fang H, Zhang H, Wu X, Chen C. Anti-inflammatory and intestinal microbiota modulation properties of Jinxiang garlic (Allium sativum L.) polysaccharides toward dextran sodium sulfate-induced colitis. J Agric Food Chem. 2020;68:12295-12309.

35. Chen Q, Wu J, Dong X, Yin H, Shi X, Su S, Che B, Li Y, Yang J. Gut flora-targeted photobiomodulation therapy improves senile dementia in an Aß-induced Alzheimer's disease animal model. J Photochem Photobiol B. 2021;216:112152.

36. Wang CS, Li WB, Wang HY, Ma YM, Zhao XH, Yang H, Qian JM, Li JN. VSL\#3 can prevent ulcerative colitis-associated carcinogenesis in mice. World J Gastroenterol. 2018;24:4254-4262.

37. Chen YJ, Wu H, Wu SD, Lu N, Wang YT, Liu HN, Dong L, Liu TT, Shen XZ. Parasutterella, in association with irritable bowel syndrome and intestinal chronic inflammation. J Gastroenterol Hepatol. 2018;33:1844-1852.

38. Kiely CJ, Pavli P, O'Brien CL. The microbiome of translocated bacterial populations in patients with and without inflammatory bowel disease. Intern Med J. 2018;48:1346-1354.

39. Wang J, Feng W, Zhang S, Chen L, Tang F, Sheng Y, Ao H, Peng C. Gut microbial modulation in the treatment of chemotherapyinduced diarrhea with Shenzhu Capsule. BMC Complement Altern Med. 2019;19:126.

40. Tu MY, Han KY, Chang GR, Lai GD, Chang KY, Chen CF, Lai JC, Lai CY, Chen HL, Chen CM. Kefir peptides prevent estrogen deficiency-induced bone loss and modulate the structure of the gut microbiota in ovariectomized mice. Nutrients. 2020;12:3432.

41. Smolensky MH, Portaluppi F, Manfredini R, Hermida RC, Tiseo R, Sackett-Lundeen LL, Haus EL. Diurnal and twenty-four hour patterning of human diseases: acute and chronic common and uncommon medical conditions. Sleep Med Rev. 2015;21:12-22.

42. Markle JG, Frank DN, Mortin-Toth S, Robertson CE, Feazel LM, Rolle-Kampczyk U, von Bergen M, McCoy KD, Macpherson AJ, Danska JS. Sex differences in the gut microbiome drive hormonedependent regulation of autoimmunity. Science. 2013;339:10841088.

43. Yurkovetskiy L, Burrows M, Khan AA, Graham L, Volchkov P, Becker L, Antonopoulos D, Umesaki Y, Chervonsky AV. Gender 
bias in autoimmunity is influenced by microbiota. Immunity. 2013;39:400-412.

44. Insenser M, Murri M, Del Campo R, Martínez-García MÁ, Fernández-Durán E, Escobar-Morreale HF. Gut microbiota and the polycystic ovary syndrome: influence of sex, sex hormones, and obesity. J Clin Endocrinol Metab. 2018;103:2552-2562.
45. Razavi AC, Potts KS, Kelly TN, Bazzano LA. Sex, gut microbiome, and cardiovascular disease risk. Biol Sex Differ. 2019;10:29.

46. Cuervo-Zanatta D, Garcia-Mena J, Perez-Cruz C. Gut microbiota alterations and cognitive impairment are sexually dissociated in a transgenic mice model of Alzheimer's disease. J Alzheimers Dis. 2021;82(s1):S195-S214. 